\title{
Pistas para la construcción de problemas en investigación cualitativa
}

Ramón Ignacio López García

Docente Universidad Nacional Autónoma de Nicaragua, Managua

nacho.ni@hotmail.com

Fecha de recibido: 6 de abril de 2015

\section{RESUMEN}

Investigar es un arte, no importa el enfoque en el que nos inscribamos para realizarlo. Sin embargo, debemos reconocer que existen prejuicios personales, que podrían obstaculizar nuestro desempeño si no tomamos medidas para desplegar nuestras capacidades. El objetivo de este escrito es invitar a la reflexión, y aclarar que construir problemas de investigación cualitativa, es una oportunidad para el aprendizaje, y no un problema en su connotación negativa. La metodología utilizada para concluir estas ideas, está centrada en la reflexión personal, la experiencia vivida como docente en asignaturas de investigación, y la crítica a los planteamientos de autores que con sus textos, pueden crear prejuicios a la comunidad investigativa sin que lo tengan premeditado.

Palabras Claves: investigación, problema, paradigmas, creatividad.
Fecha de aprobado: 12 de septiembre de 2015
Keywords: research, problem, paradigms, creativity.

\section{INTRODUCCIÓN}

En el campo de la investigación cualitativa existen dos retos a vencer, que debemos tener en constante vigilancia metodológica. Uno: construir conocimiento científico en ese ámbito no es lo mismo que para los procesos de investigación cuantitativa, su carácter humano invita a reflexionar constantemente sus formas de innovar. Dos: La creatividad necesaria en el proceso de investigaciones cualitativas, no es tan fácil desarrollar porque implica romper con procedimientos y estructuras mentales arraigadas para investigar. 
Ante ese escenario que se convierte en una preocupación metodológica, me vierte la necesidad de compartir algunas reflexiones que sirven de pistas para el desempeño metodológico en investigaciones cualitativas, y que permiten crear nuevas inquietudes sobre el tema para continuar construyendo aportes en el campo de las experiencias procedimentales investigativas, en especial, en la organización de problemas de investigación de carácter cualitativo.

Investigar es más que revisar información y elaborar una tarea necesaria en la comunidad universitaria -un estilo de vida que trasciende lo académico-. Se trata de una práctica que requiere compromiso personal y con la sociedad involucrada en nuestro tema o línea de investigación seleccionada.

En mis pocos años de experiencia docente en el campo de investigación, puedo darme cuenta que existen más barreras que elementos facilitadores en el aprendizaje, para apropiarse de los métodos de construcción de investigaciones, especialmente en las investigaciones de carácter cualitativo. Aquí es donde las inquietudes y las preocupaciones encuentran su nido, pues la intención máxima que me invade, es comunicar que cada persona que investiga, consiga la comprensión lógica del proceso de investigación. Como solemos decir, "que se enamore de su investigación", "que se enamore de lo que hace".

En el caso de investigaciones de corte cualitativo, llueven muchas interrogantes, que aprovecharemos para convertirlas en reflexiones, con el fin de encontrar pistas para la mejora en la definición de problemas de investigaciones, dado que este aspecto se reconoce como el corazón en una investigación.

Una pregunta básica es ¿Cuáles son las posibles barreras o dificultades que están afectando la comprensión y aprehensión de los nuevos conocimientos, que sobre metodología de investigación nos ofrecen para determinar problemas de investigación?

Entre las variadas respuestas a esta primera interrogante, seguramente encontremos una que nos llevará al planteamiento de esta segunda inquietud: ¿Por qué las personas no conseguimos abandonar -o nos cuesta mucho- ciertas corrientes de pensamiento que hasta hoy hemos conocido para la formulación de investigaciones? Se trata del peso de los paradigmas de la vida cotidiana que influyen constantemente en la forma en que construimos conocimiento y por efecto construimos sociedad. 
Las reflexiones descritas ahora, tratan de orientarse exclusivamente en la elaboración de Problemas de investigación con enfoque cualitativo. ¿Por qué sobre ello? Porque es esa la génesis principal de una investigación, se trata del corazón que palpitará durante todo un proceso de aplicación teórico-práctico, del que surge el nuevo conocimiento. Este conocimiento que debe trascender al conocimiento empírico.

Diremos que estas líneas no comprenden un documento concluido, ello con la esperanza de obtener reacciones en quienes tengan la oportunidad de encontrarse y enfrentarse a ellas. Reacciones que serán útiles para el enriquecimiento de las ideas, de las experiencias y del conocimiento mismo.

\section{DESARROLLO}

\section{Lo que nos dicen y lo que se rumora sobre el Problema de Investigación}

\section{“Es la investigación un problema o estamos creándonos prejuicios personales que afectan nuestra oportunidad de crear ciencia y conocimiento?"}

Iniciemos con recordar y revisar brevemente ¿qué es lo que siempre nos dicen sobre el Problema de Investigación?:

Miles de veces hemos escuchado decir a nuestros docentes y a otros estudiantes que ya han recibido asignaturas de metodología de investigación -incluso a profesionales-, expresiones como "esa clase es difícil"... “el planteamiento del problema no es fácil”... "agárrense duro que esa clase es pesada"... o bien, "jes que no sé qué es lo que quiere el profesor! Siempre me dice que no es eso el problema de investigación"... "siempre me está botando las propuestas que le muestro"...

Si hasta este momento en la lectura nos parece que estamos frente a un sentimiento bastante común, sea porque lo hemos vivido o porque lo hemos captado en otras personas, que bien, porque la intención es que podamos sentir un reflejo de esta situación. Estas expresiones encontradas en la comunidad estudiantil -y también en profesionales- sobre el planteamiento del problema de investigación son muy frecuentes, y seguramente permean la disposición de quienes se inician en procesos de investigación, haciendo de esta tarea tan importante un aprendizaje y construcción forzosa de los beneficios de la investigación. 
Por otra parte, no es para menos pensar en esas ideas de lo complejo que es esa tarea inicial de una idea de investigación. Veamos qué mensajes nos transmiten algunos autores en los libros de investigación cuando nos ofrecen sugerencias, orientaciones y la exigencia misma que debe tomarse en cuenta en la construcción de problemas de investigación.

De acuerdo con Rodrigo (1999) "cuando el investigador es novato, la etapa de la escogencia del problema ocupará gran parte del tiempo dedicado a un proyecto de investigación. Muchas veces se volverá un asunto de difícil solución" (p. 79). En adelante continua expresando que "una de las fases más difíciles de la tarea investigativa concluye cuando usted selecciona y formula correctamente el problema por investigar" (Barrantes, 1999, p. 80).

Si desde el inicio nos asumimos como nuevos en el campo de investigación, y además percibimos que el problema de investigación es algo difícil, esto podrá frenar ciertamente nuestra disposición y capacidad para desarrollarnos en el mundo investigativo. Agreguemos a esto la presión del factor tiempo -a propósito- que nos afecta en la organización de los ciclos académicos en que corresponde matricular una asignatura de investigación -en el caso de estudiantes de pregrado y posgrado-.

Por su parte, Ángeles y Munch, comparten que "una de las dificultades más grandes que afronta el investigador es plantear y delimitar el problema, ya que interrogarse adecuadamente acerca de la realidad circundante y plantearse problemas que sean relevantes, exige talento y habilidad" (Ángeles y Munch, 1993, p.38). Reforzando esos planteamientos Carlos Méndez, expresa que "la ocasión para definir un problema de cuya solución depende la de otros problemas no es un talento común entre los hombres" (Méndez, 1995, p. 62), entre personas diremos en esta lectura para incluir también al género femenino.

Obviamente, la intención de las y los autores acá citados, no es crear un prejuicio ni menos un temor en quienes realizan labor investigativa, sino, alertar para que quien investiga tome en cuenta esa realidad con autenticidad, protagonismo y compromiso. Ahora es buen momento para irse formulando otra interrogante para la identificación de pistas de apoyo a la acción de formular problemas de investigación: ¿Cuál sería una medida que podríamos tomar para enfrentar esta barrera en el proceso de investigación?... 


\section{Plantear problemas de investigación es difícil: ¿Mito o Realidad?}

"Toda dificultad que afecta nuestra dinámica personal y social, puede perfectamente ser transformada en un reto por vencer"

Parece ser que la parte práctica de plantear problemas de investigación, no es tan sencilla de acuerdo con lo que plantean varios autores, y de acuerdo con quienes han tenido la experiencia de aplicar investigaciones.

¿Será que esas ideas están calando intensamente en la capacidad de construir?, de repente es más fácil creernos las ideas negativas que las ideas positivas que nos ayudan a reforzar y ampliar nuestras capacidades para realizar procesos de investigación. ¿Es una cuestión de autoestima? Tal vez no. Pero por si acaso, debemos mantener una vigilancia constante de nuestras prácticas y plantearnos retos personales, porque las ideas negativas surten efecto en las personas siempre que no tenemos ideas positivas que nos ayuden a enfrentarlas.

\section{La creatividad como recurso personal y básico en los procesos de investigación}

"La creatividad es el arma más potente que nos permite incidir en la transformación de una realidad. El autoconcepto define nuestra decisión para utilizarla”,

La sugerencia para enfrentar esa posible dificultad mencionada, es que hay que cuidar la forma en que nos asumimos personalmente, -por si se tratase de ello-, que nadie nos alimente el prejuicio haciéndonos pensar que investigar cualitativamente -incluso investigar en general- es difícil. Por el contrario, es la experiencia más interesante donde se nos permite el uso de la creatividad y desplegar nuestras capacidades para construir.

A menos, que estemos perdiendo o nos estemos negando la posibilidad de explotar nuestras habilidades. Los tiempos en que el trabajo de investigación y ciencia eran exclusivos de la clase noble han quedado enterrados bajo las arenas de varios siglos. Actualmente, todas y todos somos seres con posibilidades de hacer ciencia a través de los procesos de investigación y obtener 
conocimiento científico como producto al usar un método sistemático y sinérgico. La investigación como tal, es uno de esos medios enriquecedores para aprender del mundo en que nos movemos.

En realidad, plantear un problema de investigación -sea de forma cualitativa o cuantitativano es difícil. Será de esa manera, si no revisamos las sugerencias proporcionadas por diferentes autores y por nuestros facilitadores académicos sobre las acciones que se deben realizar para aclarar el QUÉ se desea investigar.

Entre estas sugerencias, las más comunes son: tener interés sobre lo que se estudiará, poseer básico dominio temático, consultar con expertos en la materia, revisar documentación e investigaciones realizadas al respecto (teorías y antecedentes), y lo más importante, tener perseverancia y mucha creatividad para animarse a proponer, descubrir y construir.

\section{Construir problemas de investigación cualitativa es fácil, pero es diferente}

"La tarea de construir problemas de investigación en el campo cualitativo y cuantitativo: Ni es lo mismo, ni es igual”"

Asumamos que en este instante estamos convencidos que investigar y construir problemas de investigación NO es difícil -eso nos daría ciertas ventajas para continuar-. Ahora entonces, reflexionemos sobre una limitante que hay en el momento de construir problemas de investigación cualitativa: se trata de creer que se realiza como el caso de una investigación cuantitativa. Es curioso, pero a veces cuesta mucho trabajo sacarnos la idea que las investigaciones son algo calculado y sintetizado.

Personalmente me asusta cuando alguien me enfrenta ante una idea de problema de investigación, reduciendo éste a una pregunta. Es claro que no debe tratarse de cualquier pregunta, sino, la pregunta que guiará todo el proceso de investigación. Sin embargo, esas definiciones en el caso de investigaciones cualitativas, no son suficientes, puesto que ésta requiere acompañarse de explicaciones, de detalles, de descripciones, de argumentos y de varias interrogantes sobre el contexto en el que se desarrolla una experiencia que se desea investigar. 
Esta es la sencilla razón por la que el problema de investigación en el enfoque cualitativo no debe ser formulado en una interrogante o en la redacción de un párrafo -igual pienso sobre el caso cuantitativo-. Sería caer en el error de dejar abiertas muchas dudas y muchas interpretaciones al respecto para quien hace de lector o lectora de una propuesta de investigación. La compresión del QUÉ investigar (problema de investigación), es como se dijo antes, el corazón de una investigación. Ello exige tener claramente definida una primera imagen del paisaje por el que recorremos una geografía -la investigación-. Algunos colegas llamamos a esto: "la primer fotografía del escenario".

Para construir un problema de investigación con enfoque cualitativo, es necesario transferir a nuestro público cómo estamos comprendiendo una realidad. Sin embargo, la prevalencia del paradigma de pensamiento positivista suele estar presente, inconscientemente predominando en nuestra cultura y en nuestras ideas, interfiriendo en la posibilidad de conseguir apertura a nuevas formas de realizar investigación.

Partiendo de la corriente positivista, en investigación cuantitativa muchas personas tienden a resumir -no a reducir- el problema de investigación en una interrogante. Luego es traslada su esencia hacia un objetivo general y este a su vez, se transforma en la base para la determinación de una hipótesis que hemos de comprobar. Hasta aquí lo que nos decían sobre "es difícil", pareciera que lo cambia todo, y se nos hace más fácil reproducir lo aprendido permeado por nuestra cultura.

Es en este justo momento, donde aparece la idea que plantear un problema de investigación en el enfoque cualitativo, es de igual manera una interrogante que nos guiará en la investigación. Grave error diremos aquí, porque podemos terminar inconscientemente acostumbrándonos a elaborar una pregunta y no a construir la pregunta...

\section{No existe una norma para construir con enfoque cualitativo: ventaja aprovechable}

"La conquista de un objeto de investigación debe ser una tarea reflexiva, emergente, flexiva, pero vigilada para que no pierda criterios de calidad y esencia" 
Ahora bien, que no sea difícil construir problemas de investigación, no significa que no debamos realizar todo un esfuerzo por apropiarnos comprensivamente del QUÉ de nuestra investigación. Lo que algunos autores llaman "la conquista del objeto de investigación".

Bien cierto es, que revisando bibliografías de investigación cualitativa, no hay una receta para la elaboración de las mismas, por tanto, no hay una ley que determine cómo debe estructurarse un problema de investigación. Esto es una ventaja y una desventaja. Una ventaja porque nos permite crear y recrear la forma en que logramos conquistar y acercarnos a nuestro objeto de investigación. Una desventaja porque la incidencia y permanencia del paradigma positivista que nos provee de tradición, puede bloquear el desarrollo de nuestra creatividad y capacidad propositiva.

\section{CONCLUSIONES}

Hemos reflexionado varios aspectos sobre la realidad que vivimos quienes nos enfrentamos a la hermosa tarea de investigar incursionando en el entorno cualitativo, y lo que menos deseamos es que se piense que todo está dicho. Por el contrario, dejemos abierta este espacio para continuar compartiendo las experiencias de construcción del conocimiento. Sin embargo por ahora proponemos ciertos retos útiles para nuestras prácticas:

¿Qué tal si nos aprovechamos de esa desventaja que posee la investigación cualitativa en la que no nos presenta una receta y la transformamos en un reto?, eso resolvería fácilmente las limitaciones, pero para ello se necesita voluntad y perseverancia de quien investiga, o sea de cada uno y una de nosotros.

¿Cómo nos viene la idea de continuar practicando la creatividad y la imaginación metodológica, teórica y práctica, para alcanzar nuevas experiencias que permitan diseñar problemas de investigación relevantes en los que podemos asombrarnos hasta de las pequeños detalles de la vida cotidiana para comprender el mundo?

La invitación está abierta, al intentar nos daremos cuenta que investigar cualitativamente no solo es fácil, sino también, un proceso enriquecedor, un espacio de formación más humano y comprometido, y a la vez una emocionante aventura que permite insertarse en una realidad para aprehender de ella interactuando con el medio y todos los actores involucrados en la construcción del conocimiento. 


\section{REFERENCIAS}

Ángeles, E. y Munch, L., (1993). Métodos y técnicas de investigación. México: Trillas.

Barrantes Echavarría, R., (1999). Investigación: un camino al conocimiento. Un enfoque cuantitativo y cualitativo. San José. Costa Rica.

Méndez, C. (1995). Metodología: Guía para elaborar diseños de investigación en ciencias económicas, contables y administrativas. (2 ${ }^{\mathrm{a}}$ ed.). Colombia: Mc Graw Hill. 